\title{
ELEMENTARY PROOF OF FURSTENBERG'S DIOPHANTINE RESULT
}

\author{
MICHAEL D. BOSHERNITZAN
}

(Communicated by Andreas R. Blass)

\begin{abstract}
We present an elementary proof of a diophantine result (due to $\mathrm{H}$. Furstenberg) which asserts (in a special case) that for every irrational $\alpha$ the set $\left\{2^{m} 3^{n} \alpha \mid m, n \geq 0\right\}$ is dense modulo 1 . Furstenberg's original proof employs the theory of disjointness of topological dynamical systems.
\end{abstract}

\section{INTRODUCTION}

Throughout the paper by a semigroup we mean an infinite subset of positive integers which is closed under multiplication. Two integers $p, q$ are called multiplicitively independent if both are $\geq 2$ and the ratio of their logarithms $(\log p) /(\log q)$ is irrational. The equivalent requirement is that $p$ and $q$ should not be integral powers of a single integer.

Given a semigroup $S=\left\{s_{1}, s_{2}, \ldots\right\}, s_{1}<s_{2}<s_{3}<\cdots$, one easily verifies that the following three conditions are equivalent.

(1a) There is a pair of multiplicatively independent integers in $S$.

(1b) One cannot represent all $s_{i}$ as integral powers of a single integer.

(1c) $\lim _{i \rightarrow \infty}\left(s_{i+1} / s_{i}\right)=1$.

A semigroup is said to be nonlacunary if the above three (equivalent) conditions are satisfied.

Denote by $K$ the circle group $K=\mathbb{R} / \mathbb{Z}=[0,1)$. For an integer $n \geq 1$ a set $X \subseteq K$ is said to be $n$-invariant if $n x \in X(\bmod 1)$ whenever $x \in X$. A set $X \subseteq K$ is said to be invariant under the action of a semigroup $S$ if $X$ is $n$-invariant for every $n \in S$.

In this note we sketch an elementary proof of the following diophantine result.

Theorem 1.1 [F, Theorem 4.2]. If $S$ is nonlacunary and $\alpha$ is an irrational, then $S \alpha$ is dense modulo 1 .

The above theorem is an immediate consequence of the following. (Conversely, Theorem 1.2 easily follows from Theorem 1.1 if one takes into account Lemma 2.1 in $\S 2$; we do not use this implication.)

Received by the editors May 22, 1992 and, in revised form, December 11, 1992.

1991 Mathematics Subject Classification. Primary 11J71, 54H20.

Key words and phrases. Distribution modulo 1, topological dynamics.

The author was supported by research grant NSF-DMS-9224667. 
Theorem 1.2 [F, §4]. Let $X$ is a closed infinite subset of $K=\mathbb{R} / \mathbb{Z}=[0,1)$ which is invariant under the action of a nonlacunary semigroup $S$. Then $X=K$.

\section{Proof of Theorem 1.2}

In what follows we denote by $X^{\prime}$ the set of the limit points of $X$. We need the following two lemmas.

Lemma 2.1. Under the conditions of Theorem 1.2, assume that $X^{\prime}$ contains a rational limit point. Then $X=K$.

Lemma 2.2. Let $X$ be a closed nonempty subset of $K$ which is invariant under the action of a nonlacunary semigroup $S$. Then $X$ contains a rational point.

Theorem 1.2 follows easily from the above two lemmas. Indeed, $X^{\prime} \neq \varnothing$ (since $X$ is infinite) and satisfies all the conditions of Lemma 2.2. Therefore, $X^{\prime}$ contains a rational point, and the application of Lemma 2.1 completes the proof of Theorem 1.2. Note that Lemma 2.2 is equivalent to [F, Lemma 4.2], the lemma whose proof used the disjointness argument.

\section{Proof of Lemma 2.1}

If $0 \in X^{\prime}$, the lemma follows easily in view of (1c). For completeness, we provide the argument [F, Lemma 4.2]. Given a small $\varepsilon>0$, take $n$ such that for all $i \geq n$ the inequality $s_{i+1} / s_{i}<1+\varepsilon$ holds. Then take any $x \in X$ such that $0 \neq|x|<\varepsilon / s_{n}$, and observe that the finite set

$$
\left\{s x\left|s \in S, s_{n} \leq s \leq 1 /\right| x \mid\right\} \subset X
$$

is $\varepsilon$-dense in $K$. Since $\varepsilon>0$ is arbitrary and $X$ is closed, $X=K$.

Now assume that $X^{\prime}$ contains a rational $r=n / t$. Take a pair $p, q$ of multiplicatively independent integers of $S$ (which exists since $S$ is nonlacunary). Without loss of generality, we assume that $(n, t)=(t, p)=(t, q)=1$ (replacing if needed $r$ by the product of $r$ and suitable powers of $p$ and $q$; $(x, y)$ denotes the greatest common divisor of $x$ and $y)$. Choose a positive integer $u$ such that $p^{u} \equiv q^{u} \equiv 1(\bmod t)$ (e.g., $u=\phi(t)$ where $\phi$ is the Euler function). The sets $X$ and $X^{\prime}$ are clearly both $p^{u}$ - and $q^{u}$-invariant, and so are their shifts $Y^{\prime}=X^{\prime}-r$ and $Y=X-r$, in view of the choice of $u$. Moreover, $0 \in Y^{\prime}$. Applying the conclusion of the preceding paragraph to $Y$ and $Y^{\prime}$, we conclude that $Y=K$. Therefore, $X=K$.

\section{Proof of Lemma 2.2}

Assume that $X$ does not contain rationals. Let $\varepsilon>0$ be given. First choose a multiplicatively independent pair $p, q \in S$. Next choose an integer $t \geq 3$ such that $\varepsilon t>1,(t, p)=1$, and $(t, q)=1$. Finally, choose a positive integer $u$ such that $p^{u} \equiv q^{u} \equiv 1 \quad(\bmod t)$.

Define (inductively) a sequence of sets

$$
X=X_{0} \supseteq X_{1} \supseteq \cdots \supseteq X_{t-1}
$$

by assuming

$$
X_{i+1}=\left\{x \in X_{i} \mid x+1 / t \in X_{i}(\bmod 1)\right\} \quad \text { for } 0 \leq i \leq t-2 .
$$


Observe that, for every $X_{i}, 0 \leq i \leq t-1$, the following properties hold:

(a) $X_{i}$ is both $p^{u}$ - and $q^{u}$-invariant.

(b) $X_{i}$ is closed in $K$.

(c) $X_{i}$ is an infinite set of irrational numbers.

Indeed, (a), (b), and (c) clearly hold for $i=0$. We proceed by induction.

Assume that these properties take place for some $i, 0 \leq i \leq t-2$; we have to validate the properties for $i+1$. Consider the set $D_{i}$ of the differences in $X_{i}$ :

$$
D_{i}=X_{i}-X_{i} \subseteq K \text {. }
$$

$D_{i}$ is a closed subset of $K$ (because $X_{i}$ is compact) which is both $p^{u}$ - and $q^{u}$-invariant, and 0 must be its limit point (since $X_{i}$ is infinite). The semigroup generated by $p^{u}$ and $q^{u}$ is nonlacunary, and therefore $D_{i}=K$ (Lemma 2.1). It follows that the set $X_{i+1}$ (defined by (4.1)) is nonempty.

Moreover, $X_{i+1}$ is both $p^{u}$ - and $q^{u}$-invariant (in view of the choice of $u$ ) and consequently is infinite. Finally, $X_{i+1}$ is closed in $K$ because $X_{i}$ is.

It follows that $X_{t-1} \neq \varnothing$. Take $x_{0} \in X_{t-1}$, and let $x_{i}=x_{0}+i / t, 0 \leq i \leq$ $t-1$. The set $A=\left\{x_{i} \mid 0 \leq i \leq t-1\right\}$ is $\varepsilon$-dense in $K$ (since $1 / t<\varepsilon$ ), and $A \subseteq X=X_{0}$ (in fact, $x_{i} \in X_{t-i-1}$ ). Since $\varepsilon>0$ is arbitrary, $X$ is dense in $K$, and hence $X=K$, a contradiction with the assumption that $X$ does not contain rationals.

\section{SOME RELATED RESULtS}

For a sequence $\overline{\mathbf{s}}=\left\{s_{i}\right\}_{i \geq 1}$ of reals denote

$$
\operatorname{NUD}(\overline{\mathbf{s}})=\left\{\alpha \in \mathbb{R} \mid\left\{\alpha s_{i}\right\} \text { is not } u \text { niformly distributed in } K\right\}
$$

and

$$
\mathrm{ND}(\overline{\mathbf{s}})=\left\{\alpha \in \mathbb{R} \mid\left\{\alpha s_{i}\right\} \text { is not } d \text { ense in } K\right\}
$$

(with the numbers $\alpha s_{i}$ considered mod 1, i.e., as the elements of $K$ ). Clearly, for every $\overline{\mathbf{s}}$ the inclusion $\mathrm{ND}(\overline{\mathbf{s}}) \subseteq \mathrm{NUD}(\overline{\mathbf{s}})$ takes place.

A classical theorem by Weyl asserts that the Lebesgue measure of $\mathrm{NUD}(\overline{\mathbf{s}})$ is 0 for any sequence $\overline{\mathbf{s}}$ of distinct integers. If $s_{i+1} / s_{i}>1+\varepsilon$ for some $\varepsilon>0$ and all large $i$, the set $\mathrm{ND}(\overline{\mathbf{s}})$ has Hausdorff dimension 1 and, in particular, is uncountable [P, M1, M2]. On the other hand, we have proved recently [Bos2] that if $\overline{\mathbf{s}}$ is an unbounded sequence of positive reals such that $\lim _{i \rightarrow \infty}\left(s_{i+1} / s_{i}\right)=$ 1 (such sequences are called sublacunary), then the Hausdorff dimension of $\mathrm{ND}(\overline{\mathbf{s}})$ is 0 . (A sharp upper bound on the Hausdorff dimension of the set $\mathrm{NUD}(\overline{\mathbf{s}})$ in terms of the polynomial growth of the sequence $\overline{\mathbf{s}}$ is given in [ET, Theorem 13].)

Note that for most (in a certain probability sense) of the sequences $\overline{\mathbf{s}}$ of integers having a prescribed subexponential asymptotic growth we have $\operatorname{NUD}(\overline{\mathbf{s}})=$ $\mathbb{Q}$, the field of rational numbers (see [Bos1, AHK, Bou]).

On the other hand, if $\overline{\mathbf{s}}$ is the sequence of all elements of a semigroup (of integers) taken in the increasing order which grows faster than polynomials

$$
\limsup _{i \rightarrow \infty} \frac{\log s_{i}}{\log i}=\infty \text {, }
$$

then $\operatorname{NUD}(\overline{\mathbf{s}})$ is uncountable. The requirement of transpolynomial growth cannot be weakened; for any fixed integer $n \geq 1$ we have $\operatorname{NUD}(\overline{\mathbf{s}})=\mathbb{Q}$ for 
$\overline{\mathbf{s}}=\left\{i^{n}\right\}$. Under the stronger condition that the semigroup is finitely generated, the set $\operatorname{NUD}(\overline{\mathbf{s}})$ has Hausdorff dimension 1 [Be5].

Note that our proof of Furstenberg's theorem is close in spirit to some of the constructions of Berend [Be3] where analogous results were obtained for so-called multiplicative $I P$-sets of integers. Berend also dealt with the multidimensional case [Be1, Be2] and with multiplicative semigroups of algebraic numbers [Be4].

\section{REFERENCES}

[AHK] M. Ajtai, I. Havas, and J. Komlós, Every group admits a bad topology, Stud. Pure Math., Memory of P. Turan, Birkhäuser, Basel and Boston, 1983, pp. 21-34.

[Be1] D. Berend, Multi-Invariant sets on tori, Trans. Amer. Math. Soc. 280 (1983), 509-532.

[Be2] _ Multi-Invariant sets on compact abelian groups, Trans. Amer. Math. Soc. 286 (1984), 505-535.

[Be3] _ Actions of sets of integers on irrationals, Acta Arith. 48 (1987), 275-306.

[Be4] _ Dense $(\bmod 1)$ dilated semigroups of algebraic numbers, J. Number Theory 26 (1987), 246-256.

[Be5] _ private communication.

[Bos1] M. Boshernitzan, Homogeneously distributed sequences of integers of sublucunary growth, Monatsh. Math. 96 (1983), 173-181.

[Bos2] _ Density modulo 1 of dilations of sublacunary sequences, Adv. Math. (to appear).

[Bou] J. Bourgain, On the maximal ergodic theorem for certain subsets of the integers, Israel J. Math. 61 (1988), 39-72.

[ET] P. Erdös and S. J. Taylor, On the set of points of convergence of a lacunary trigonometric series and the equidistribution properties of related sequences, Proc. London Math. Soc. (3) 7 (1957), 598-615.

[F] H. Furstenberg, Disjointness in ergodic theory, minimal sets, and a problem in diophantine approximation, Math. Systems Theory 1 (1967), 1-49.

[M1] D. de Mathan, Sur un probleme de densite modulo 1, C. R. Acad. Sci. Paris Sér. I Math. 287 (1978), 277-279.

[M2] , Numbers contravening a condition in density modulo 1, Acta Math. Hungar. 36 (1980), 237-241.

[P] A. D. Pollington, On the density of sequence $\left\{n_{k} \theta\right\}$, Illinois J. Math. 23 (1979), 151-155.

Department of Mathematics, Rice University, Houston, Texas 77251

E-mail address: michael@math.rice.edu 